\title{
Changes in Pluto's Atmosphere
}

\author{
J. L. Elliot \\ Dept. of Earth, Atmospheric, and Planetary Sciences, MIT, Cambridge, \\ MA 02139 Dept. of Physics, MIT and Lowell Observatory, Flagstaff, \\ $A Z 86001, U S A$
}

Pluto's tenuous atmosphere was probed in 1988 with a stellar occultation observed from the Kuiper Airborne Observatory (KAO, Elliot et al. 1989) and a variety of ground-based sites (Millis et al. 1993). These data, subsequent theoretical modeling (e.g. Strobel et al. 1996), and spectroscopic observations (Owen et al. 1993; Young et al. 1997), gave us the following post-occultation picture of Pluto's atmosphere (Yelle \& Elliot 1997; Elliot, Person, \& Qu 2003b): $\mathrm{N}_{2}$ is the dominant constituent of the atmosphere, which also contains small amounts of $\mathrm{CH}_{4}$ and $\mathrm{CO}$. These molecules are in vapor-pressure equilibrium with their surface ices, and this process acts as a thermostat to keep the $\mathrm{N}_{2}$ ice at $\sim 38$ $\mathrm{K}$ around the body. The temperature of the 1-3 $\mu$ bar pressure region probed by the occultation was $\sim 100 \mathrm{~K}$. The $\mathrm{KAO}$ light curve dropped abruptly, however, just below half-light. This abrupt drop could be due to one of two potential properties of Pluto's atmosphere: extinction, or a steep thermal gradient. Each of these explanations has strengths and weaknesses (Yelle \& Elliot 1997; Elliot, et al. 2003b).

Pluto's atmosphere was probed again with two stellar occultations in 2002 for the first time since 1988-with the twofold goals of resolving the extinction vs. thermal-gradient issue and to look for possible changes over time. Data for the first event were recorded with two small telescopes and showed that Pluto's atmosphere had changed (Buie et al. 2002; Sicardy et al. 2003), but the data quality was not sufficient for further conclusions. Observations of the second event provided more extensive results. Data were recorded from nine telescopes, located in Hawaii, Arizona, and California at both visible and IR wavelengths (Elliot et al. 2003a). The most striking result was the marked difference between the shape of the occultation light curves observed in 1988 and 2002 (Elliot, et al. 2003a; Sicardy, et al. 2003). Sharp light-curve spikes were observed, which were barely detected in 1988. The spikes indicate dynamical activity in Pluto's atmosphere-caused either by waves, turbulence, or a combination of both. Based on the astrometric solution for the occultation (as derived from the light-curve timings from the different observing sites), it was determined that the atmospheric pressure had increased about a factor of two. For most of this time Pluto has been receding from the sun (perihelion occurred in 1989). The most likely cause for this pressure increase appears to be a warming of the $\mathrm{N}_{2}$ surface ice by about $1 \mathrm{~K}$, which would then cause the requisite pressure increase in order to maintain equilibrium. Surface warming could be due to the effects of thermal inertia or a small increase in the amount of insolation absorbed by the surface ice (e. g. Hansen \& Paige 1996). 
Another result is an observed trend of decreasing depth of the occultation with increasing wavelength of observation, between 0.7 and $2.2 \mu \mathrm{m}$ (Elliot et al. 2003a). This trend is consistent with extinction from micron-sized particles in Pluto's atmosphere. As yet we cannot rule out the trend being caused by the residual flux from a faint, red companion star that was not occulted. Observations of the occulted star with adaptive optics (Liu, personal communication) have not yet indicated the presence of such a companion, but a more extensive search will be required to completely resolve the issue.

More occultation opportunities to probe Pluto's atmosphere will occur in the next few years, as Pluto approaches alignment with the galactic plane (McDonald \& Elliot 2000). Some of these events can be observed simultaneously in the visible and IR (Dunham, Elliot, \& Taylor 2000) when the Stratospheric Observatory for Infrared Astronomy (SOFIA) becomes operational. Pluto's atmosphere will be probed by spacecraft as early as 2015, with the flyby of the New Horizons mission (Stern \& Spencer 2003).

This work was funded at MIT in part by NASA Grant NAG5-10444 and NSF Grant AST-0073447.

\section{References}

Buie, M. W., et al. 2002, Bull. Amer. Astron. Soc., 34, 877

Dunham, E. W., Elliot, J. L., \& Taylor, B. W. 2000, Proc. S.P.I.E., 4014, 76

Elliot, J. L., et al. 2003a, Nature, 424, 165

Elliot, J. L., Dunham, E. W., Bosh, A. S., Slivan, S. M., Young, L. A., Wasserman, L. H., \& Millis, R. L. 1989, Icarus, 77, 148

Elliot, J. L., Person, M. J., \& Qu, S. 2003b, Astron. J., 126, 1041

Hansen, C. J., \& Paige, D. A. 1996, Icarus, 120, 247

McDonald, S. W., \& Elliot, J. L. 2000, Astron. J., 119, 1999

Millis, R. L., et al. 1993, Icarus, 105, 282

Owen, T. C., et al. 1993, Science, 261, 745

Sicardy, B., et al. 2003, Nature, 424, 168

Stern, S. A., \& Spencer, J. 2003, Earth, Moon, and Planets, (in press)

Strobel, D. F., Zhu, X., Summers, M. E., \& Stevens, M. H. 1996, Icarus, 120, 266

Yelle, R. V., \& Elliot, J. L. 1997, in Pluto and Charon, eds. S. A. Stern, \& D. J. Tholen (Tucson, AZ: Univ. of Arizona Press), 347

Young, L. A., Elliot, J. L., Tokunaga, A., de Bergh, C., \& Owen, T. 1997, Icarus, 127, 258 